\title{
Genomic and postgenomic diversity of fungal plant biomass
} degradation approaches

\section{de Vries, Ronald}

2020-06

de Vries , R \& Mäkelä , M R 2020 , ' Genomic and postgenomic diversity of fungal plant biomass degradation approaches ' , Trends in Microbiology , vol. 28 , no. 6 , pp. 487-499 . https://doi.org/10.1016/j.ti

http://hdl.handle.net/10138/326254

https://doi.org/10.1016/j.tim.2020.01.004

cc_by_nc_nd

acceptedVersion

Downloaded from Helda, University of Helsinki institutional repository.

This is an electronic reprint of the original article.

This reprint may differ from the original in pagination and typographic detail.

Please cite the original version. 
3 Ronald P. de Vries ${ }^{1 *}$, Miia R. Mäkelä²

$4{ }^{1}$ Fungal Physiology, Westerdijk Fungal Biodiversity Institute \& Fungal Molecular Physiology,

5 Utrecht University, Utrecht, The Netherlands; ${ }^{2}$ Department of Microbiology, University of

6 Helsinki, Helsinki, Finland.

7 Correspondence: $\underline{\text { r.devries@wi.knaw.nl }}$

$9 \quad$ Keywords

10 Fungi; plant biomass degradation; regulation; CAZy; enzymes

12 Abstract

13 Plant biomass degradation by fungi is a widely studied and applied field of science, due to its 14 relevance for the global carbon cycle and many biotechnological applications. Before the 15 genome era, many of the in-depth studies focused on a relatively small number of species, whereas now, many species can be addressed in detail, revealing the large variety in the

17 approach used by fungi to degrade plant biomass. This variation is found at many levels and 18 include genomic adaptation to the preferred biomass component, but also different 19 approaches to degrade this component by diverse sets of activities encoded in the genome. 20 Even larger differences have been observed using transcriptome and proteome studies, even 21 between closely related species, suggesting a high level of adaptation in individual species. A 22 better understanding of the drivers of this diversity could be highly valuable in developing more 23 efficient biotechnology approaches for the enzymatic conversion of plant biomass. 


\section{Composition of plant biomass and its enzymatic degradation}

Plant cell walls are the major fraction of plant biomass and mainly consist of polysaccharides (cellulose, hemicellulose, pectin), the aromatic polymer lignin and proteins [1]. Plant biomass also contains storage polysaccharides, such as starch, inulin and several gums. The type, relative amount and structure of these polymers varies considerably, depending on plant species, tissue, age and growth season, and they consist of different monomeric building blocks. This diversity generates the need for a broad spectrum of enzymes to efficiently degrade plant biomass. The fungal enzymes involved in the degradation process have been reviewed extensively before [1-4] and will not be discussed in detail here. Plant biomassdegrading enzymes (with the exception of most feruloyl esterases) have been catalogued in the Carbohydrate Active enZYme database (www.cazy.org) [5] in families according to their amino acid sequence similarity. Many of these families have been shown to contain multiple enzyme activities, which has led to the establishment of subfamilies for some of them, reflecting individual enzyme activities [6-8]. CAZy (see Glossary) annotation has become a standard feature of all fungal genomes included in the MycoCosm database [9], enabling a quick comparison of the putative plant biomass degrading abilities of the corresponding species. However, the lack of available characterized enzymes in many of the CAZy families can result in mis-predictions of their degradation ability (Box 1).

Efficient degradation of plant biomass requires not only a broad set of enzymes, but also efficient synergy of their activities. Synergy in the degradation of plant polysaccharides has been studied in detail, demonstrating that simultaneous activity of the enzymes leads to a higher release of monosaccharides than sequential activity $[10,11]$. This synergy is not restricted to the positive effect of combining a backbone and side-chain cleaving enzyme, but also occurs between different side-chain cleaving enzymes [12]. Efficient lignin degradation requires the combined action of lignin-oxidizing peroxidases and $\mathrm{H}_{2} \mathrm{O}_{2}$-generating enzymes [13]. In recent years, the benefits of enzyme synergy have also been used for biotechnological applications [14-19], demonstrating that combining enzymes from different fungal species can benefit the overall biomass hydrolysis [20]. 


\section{Different life styles and biotopes require different approaches to plant biomass degradation}

Plants and plant biomass are of major importance for many fungi, as they are a major source of carbon in terrestrial biotopes. The life styles of fungi strongly affect how and to which extent they degrade plant biomass. Plant biomass degrading fungi are found throughout the fungal kingdom, but most studies have focused on ascomycetes and basidiomycetes. Plant biomass degradation has been studied in most detail in saprobic fungi, in part because they are the species of choice for biotechnology [21]. Saprobic fungi typically have large arsenals of plant biomass-degrading enzymes (see below) to degrade their dead and sometimes already decaying substrate.

Among the wood-degrading basidiomycetes, a further distinction can be made, which was traditionally based on the visual appearance of the degraded wood [22, 23]. White-rot fungi degrade both lignin and polysaccharides, using a broad enzymatic arsenal, while brown-rot fungi only modify lignin but efficiently degrade (hemi-)cellulose, using a more limited set of enzymes together with Fenton chemistry. In recent years several species have been identified that have characteristics of both white- and brown-rot fungi, which are referred to as grey-rot fungi [24].

Plant pathogenic fungi have the additional challenge of overcoming the host defense system to be able to penetrate the host tissue and gain access to their carbon source [25]. The process of plant biomass degradation after penetration can differ significantly, covering biotrophic (e.g. Ustilago maydis [26]) to necrotrophic (e.g. Alternaria brassicicola [27]) life styles. It has been shown for various plant pathogens that specific plant biomass-degrading enzymes play a role in the penetration of the plant tissue, while a broader set of plant biomass-degrading enzymes is produced during the necrotrophic/degradation phase of their life cycle [28].

Symbionts, such as mycorrhizal fungi, cause typically only limited damage to the plant cell walls, to allow the establishment of connections between the mycelium and the plant roots, but to prevent significant weakening of their host [29]. 


\section{Insights from fungal genomes into diversity in plant biomass degradation}

82 The different fungal life styles are often well-reflected in the set of genes encoding plant

83 biomass-degrading enzymes present in their genomes and often show correlations to the natural substrate of the species. Examples of this are an increase in enzymes acting on cellulose

85 and xylan in the cereal pathogen Pyricularia (Magnaporthe) oryzae [30], while Botrytis cinerea [31], a common pathogen of strawberry and tomato, contains a high number of pectinases in

87 its genome. A comparison of growth profiles of $>400$ fungal species/strains on plant biomass related substrates (www.fung-growth.org) to their genome content revealed that for most species, the increase or decrease in putative enzymes related to degradation of a specific polymer correlates well with the ability of a fungus to use that polymer as a carbon source. An example of that is given for two fungi in Fig. 1, where the number of putative xylanolytic and pectinolytic genes correlates well with the ability of these species to grown on xylan or pectin.

Figure 1. Comparison of growth of two ascomycete fungi on xylan and pectin, and their monomeric components (www.fung-growth.org), and the number of putative xylanolytic and pectinolytic genes in their genome [31, 32]. The CAZy families that were taken into account in this comparison are listed. $\mathbf{G H}=$ glycoside hydrolase, $\mathbf{P L}=$ polysaccharide lyase, $\mathbf{C E}=$ carbohydrate esterase.

There are some notable exceptions to this correlated pattern, such as the commonly used industrial cellulase producer Trichoderma reesei [33]. The high ability of $T$. reesei to degrade

103 a limited set of cellulases [34].

104 However, while this correlation of growth and overall CAZy genome content is common, this 105 does not apply when the number of genes in individual CAZy families are evaluated. The 106 increasing number of sequenced fungal genomes have highlighted high diversity in the numbers 
of genes in specific CAZy families across the fungal kingdom. These differences suggest differences at several levels. As mentioned above, adaptation to specific plant polysaccharide can be observed, but also different approaches for degradation of specific polysaccharides. One of the best examples of this is degradation of pectin by different fungal species. Among the Aspergilli, the Aspergillus niger genome contains mostly pectin hydrolases, while Aspergillus nidulans and Aspergillus oryzae have a higher number of pectin lyases and a lower number of pectin hydrolases [35]. A. niger acidifies its medium, while the other two species do not, which fits with the preference for acid conditions for pectin hydrolases and neutral conditions for pectin lyases. A similar difference was observed for Myceliophthora thermophila and Thielavia terrestris, with the first being rich in pectin lyases and poor in pectin hydrolases and the opposite for the second species [36]. The functional implications of this were also confirmed, as M. thermophila grew better on pectin at neutral to alkaline $\mathrm{pH}$, while $T$. terrestris grew best at acidic $\mathrm{pH}$. However, difference in approach can also be observed for other polysaccharides. The relative number of $\mathrm{GH} 10$ and $\mathrm{GH} 11$ endoxylanases can differ strongly in fungal genomes (e.g. Table 1, Aspergillus carbonarius and Aspergillus wentii). GH10 endoxylanases are more active on branched xylans, while GH11 act mainly on non-branched stretches in xylan [37], resulting not only in different product profiles, but also affecting the overall ability of these fungi to degrade different xylans.

These differences are also observed in closely related species that have similar habitats. Several examples of this are given in Table 1, demonstrating significant differences in numbers of genes per CAZy family between species of the same genus. Penicillium subrubescens stands out from the other Penicillia for the strong expansions in specific CAZy families (e.g. GH11, GH28, GH43) [38]. Similarly, Trichoderma atroviride has a higher number of $\mathrm{GH} 43$ genes than the other Trichoderma species, while Phanerochaete chrysosporium has a higher number of cellulose degradation related GH7 and GH74 genes than Phanerochaete carnosa (Table 1). Talaromyces marneffei has a reduced set of CAZy genes compared to the other species of this genus (Table 1), possibly due to its modified life style as a pathogen of mammals [39]. In fact, species-specific expansions of certain CAZy families are relatively common [4, 38, 40] and likely reflect an increased need for the related activity. In some cases, it has been shown that these closely 
related species co-inhabit the same biotope, which could suggest that the different species in the same habitat evolved to focus primarily on different components of the biomass, thereby reducing the direct competition between them. Support for this can in particular be found in fungal genera for which many species have been genome-sequenced, such as Aspergillus (Box 2).

Table 1. Comparison of the number of genes in selected CAZy glycoside hydrolase (GH) families related to plant biomass degradation in species of the same genus. Data was based on the CAZy annotation tables of published genomes in MycoCosm [9]. Expanded gene numbers are in bold.

Another level of diversity can be found in the fungal ability to degrade different types or components of plant biomass, differentiating the more generalist fungi, with a broad biotope and/or substrate range, from the more specialist fungi. While there is a large grey-area between these two extremes, the differences can easily be seen when comparing some example species. The generalist ascomycete fungus $A$. niger possesses a broad spectrum of plant biomass-degrading enzymes [41], but the numbers per CAZy family are average compared to other fungi. In contrast, Podospora anserina is an ascomycete fungus that only lives in herbivore dung as a late colonizer, during which only highly complexed lignin, cellulose and xylan is present. The genome of $P$. anserina contains a strongly increased number of cellulases and xylanases, but a much reduced number of pectinases, compared to A. niger and other generalist fungi [32]. This is also reflected in its ability to grow on different plant polysaccharides (Fig. 1). More detailed analysis of these differences may enable predictions of genome content based on biotope range.

\section{Post-genomic diversity in plant biomass degradation}

To address the reason for the huge numbers of (putative) enzymes in fungal genomes related to plant biomass degradation, many transcriptome and proteome studies during growth of 
fungi on plant biomass have been reported. In most cases these studies revealed a tailored response to the composition of the biomass the fungus was exposed to, demonstrating the ability of fungi to adapt to the prevailing substrate. This adaptation was clearly shown during growth of the thermophilic ascomycete $M$. thermophila on a range of mono- and dicot substrates [42]. Only a small set of genes was induced on all plant biomass substrates, with more specific sets observed for the individual substrates. Interestingly though, the adaptation response does not seem to be restricted to substrates a fungus encounters in its natural habitat. When the wood-degrading white-rot basidiomycete Dichomitus squalens was grown on wood and non-woody biomass, it also adapted efficiently to the non-woody substrates (wheat bran and cotton seed hulls) [43], suggesting that its ability exceeds its natural substrates. This broader ability of fungi can be explored in biotechnology for the production of efficient enzyme cocktails, especially when the right substrate has been selected for the production of the enzyme cocktail. A comparison of the production of plant biomass-degrading enzymes by $P$. anserina on three plant biomass revealed that the most diverse enzyme mixture was produced on soy bean hulls and this mixture resulted in higher saccharification of all three biomass [44]. Interestingly, the tailored transcriptomic response of fungi to different substrates seems to be a major factor in the diversity of the plant biomass degrading approach of fungi. Comparison of the response of basidiomycete fungi in 22 plant biomass related transcriptome datasets, revealed a narrow set of conserved genes that are commonly expressed during growth on plant biomass, while the majority of the genes involved in plant biomass degradation responded only in some species or conditions [45]. The extensive number of studies addressing plant biomass degradation by fungi suggests that each species has a modified approach, when examined at the molecular level, both with respect to genome content of genes encoding plant biomassdegrading enzymes and the set of expressed genes in the presence of a certain (component of) plant biomass. In some cases, these differences are related to preferred environmental conditions. As mentioned earlier, comparison of $M$. thermophila to the related species $T$. terrestris demonstrated a difference in pectinolytic genes that could be directly related to their ability to degrade pectin at different $\mathrm{pH}$ [36]. This study also revealed significant variation in the set of genes that was expressed when these two fungi were exposed to the same substrate. A 
similar difference in transcriptomic and/or proteomic response has even been observed between closely related species, such as in the genus Aspergillus (Box 2).

\section{Regulation of genes encoding plant biomass-degrading enzymes}

A combination of enzymatic activities is required to degrade the complex structure of plant biomass and therefore fungi typically produce diverse enzyme mixtures during growth on plant biomass. Co-regulation of genes encoding enzymes acting on the same plant polymer, but also enzymes acting on different plant polymers has been reported across the fungal kingdom [42, 46-50]. A detailed transcriptomic study in A. niger using both wild type and regulatory mutants grown on various plant biomass related sugars, revealed extensive co-regulation of genes encoding enzymes acting on different plant biomass polysaccharides [51]. Co-expression of genes acting on different components of plant biomass has also been observed for several wood-degrading basidiomycete fungi [46, 48-50] and several anaerobic fungi [47]. One reason for the co-regulation of these genes could be that by already producing some enzymes for a secondary polymer, the fungus is preparing to degrade it, once the primary substrate has been largely consumed. In addition, the complex structure of plant biomass may require partial degradation of some polymers, in order for the fungus to gain access to the polymer it focusses on as its main substrate.

So far, the molecular regulation of plant biomass degradation by fungi has been mainly studied in ascomycete fungi, including the identification of several regulators [52]. Overall, the regulatory system typically consists of a number of transcriptional activators, each responding to their own monomeric or dimeric inducer, the general carbon catabolite repressor protein CreA/CRE1, and some more specific transcriptional repressors. Except for CreA/CRE1, none of these regulators are conserved across the fungal kingdom. Some (XInR/XLR1, CLR1/ClrA, CLR2/ClrB/ManR) are present in most filamentous ascomycetes [52], albeit with variation in their target gene set (see Box 3). However, none of the transcriptional activators of ascomycetes appear to have orthologs in basidiomycetes, despite conservation of the enzyme sets and the conditions under which the enzymes are produced (see above). This suggests that 
the regulatory systems have evolved after the split of these two phyla. Also within the ascomycetes, differences in the presence of regulators can be observed [52], including one clear example of parallel evolution. Two L-arabinose responsive transcriptional activators have been identified, one in the Eurotiomycetes (AraR) [53] and one in the Sordariomycetes and Leotiomycetes (Ara1) [54, 55]. Both activators control the expression of a very similar set of genes involved in release of L-arabinose from plant biomass and the subsequent intracellular conversion of this sugar, but share no significant sequence similarity [55]. Interestingly, neither of these regulators has an orthologue in Dothidiomycete fungi, suggesting that they either do not have an L-arabinose specific regulator or that the regulator with this function is not related to AraR or ARA1.

Evidence for interaction of regulators has also been reported, but the molecular basis of this interaction has remained largely unknown. As most regulators control the expression of genes encoding enzymes that release a variety of monomers (see above) and these monomers themselves result in the activation of other regulators, a system of interactive activation of gene expression balanced with a general repression via CreA/CRE1 can be envisioned (Fig. 2), in addition to more direct interactions.

Figure 2. Model for the indirect interaction between regulators involved in plant biomass degradation in A. niger, based on [51]. The transcriptional activators facilitate the release of various monomers from plant biomass (indicated by thin arrows). These monomers themselves result in activations of specific regulators (indicated by thick arrows). CreA has an overruling effect in that high concentrations of any of the monomers will repress plant biomass degradation.

CreA/CRE1 has been shown to have a major impact on the production of plant biomassdegrading enzymes $[52,56]$, and in fact was lost in the hypercellulolytic $T$. reesei mutant RUTC30 [57]. While there is no evidence for direct interaction between CreA/CRE1 and other regulatory proteins involved in plant biomass degradation, it has been shown in $A$. niger that 
248 the expression level of an XInR-regulated gene is the result of the balance between induction by $249 X \ln R$ and repression by CreA [58]. The induction through XInR seems to be independent, while 250 the repressing effect through CreA is clearly dependent on the D-xylose concentration [58]. In 251 addition, it was also shown that all monomeric sugars can cause CreA-mediated repression, but 252 that the strength of this repression depends on the individual monosaccharide, with D-glucose 253 and D-xylose causing the strongest repression in A. niger and L-rhamnose the weakest [59]. This 254 correlates with the order in which sugars are taken up by this species, when they are present as 255 a mixture, but surprisingly this sequential uptake was not affected in a CreA mutant [60]. Interaction between other factors, not involving CreA, has also been reported. In Neurospora 257 crassa and other Sordariomycetes, crosstalk between cellulose and mannan perception 258 involved the main transcriptional regulator CLR-2, but also suggested a role for import of 259 manno- and cellodextrins, and possibly other regulators [61]. In A. niger an antagonistic effect between the two pentose-related transcriptional activators, AraR and XInR, was reported [53]. XInR target genes are normally induced on D-xylose, while AraR target genes are induced on Larabinose in $A$. niger. However, deletion of $x \ln R$ results in reduced expression of its target genes on D-xylose, while the expression of the AraR target genes increases on this sugar. Similarly, deletion of araR results in reduced expression of its target genes, but increased expression of $\mathrm{X} \ln \mathrm{R}$ target genes on L-arabinose. The molecular mechanism underlying this effect is not known, but this does not appear to be caused by direct regulation of $x \ln R$ or $\operatorname{ara} R$ by the other regulator as there are no indications that these transcriptional activators can also act as repressors. AraR and XInR were also shown to co-regulate the D-galactose oxido-reductive pathway in A. nidulans together with the D-galactose responsive transcriptional activator GalR [62]. Similarly, degradation of pectin and conversion of the released monomers is dependent 271 on at least three transcriptional activators in A. niger, GaaR, RhaR and AraR [63]. However, 272 these studies only scratch the surface of the interaction in the complex regulatory network that controls plant biomass degradation in these fungi. More detailed studies are required to fully

274 reveal the molecular basis of the hierarchy and interaction of the individual regulators involved 275 in this process. Considering the highly similar expression profiles observed for genes involved in 276 plant biomass degradation in basidiomycetes, it can be assumed that a similar complex 
277 regulatory network also exists in these fungi. The absence of clear homologs of the ascomycete 278 regulators in basidiomycetes (with the exception of CreA/CRE1 [64]) suggests that this network 279 is built from different regulators, possibly from different regulator classes. The majority of the ascomycete transcriptional activators are members of the $\mathrm{Zn}_{2} \mathrm{Cys} 6$ class of regulators, which is the most expanded class in ascomycetes [65]. In basidiomycetes, the CCHC class of regulators is clearly expanded, which may indicate that these proteins could contain several of the plant biomass related regulators.

\section{Concluding remarks}

Plant biomass degradation by fungi is a highly complex process that is not only of major importance for the life of many fungi and the global carbon cycle, but also has a long history of use in biotechnological applications in the food \& feed, pulp \& paper and biofuel \& biochemical industries. The availability of an increasing number of fungal genomes has revealed the large diversity of genes encoding plant biomass-degrading enzymes that fungi have as well as the 291 large differences in these gene sets between the species. Fungal genomes also demonstrated 292 that all species appear to have a modified approach and that model species do not really exist 293 for this process. The classical 'model' fungus for cellulose degradation, T. reesei, was in fact 294 shown to have a highly unusual approach to plant biomass degradation (see above) [34] and 295 therefore does not represent the majority rule used by other fungi. It would therefore be better 296 to avoid the word 'model species' in this field and rather use the term 'reference species', 297 which together can reflect the diversity of this process.

298 The regulation of gene expression appears to have a dominant effect on diversity of plant 299 biomass degradation approaches of fungi. Even related fungi with similar genome content 300 produce highly diverse enzyme sets when grown on the same plant biomass substrate [66]. This 301 has so far not been studied across the fungal kingdom as there are only few fungal genera with 302 an available genome sequence for multiple species, and plant biomass degradation is studied in 303 only some of them. While an increasing number of regulators involved in this process is being 
identified in ascomycete fungi, almost none have been identified in other fungal phyla, severely limiting our understanding.

Genomic and post-genomic comparisons of fungi are typically based on a single isolate of a species, as for most species only one genome sequence is available. This raises questions whether these comparisons truly reveal differences between species, as this is largely dependent on the diversity within a species, and to which extent the selected strain is a good representative of the species. In most cases, no extensive comparison of strains has been performed before selection of the strain for genome sequencing. Often (e.g. A. nidulans [67]), the sequenced strain has been domesticated for many decades and may no longer be very similar to isolates recently obtained from natural habitats. When multiple strains were genome sequenced, clear differences were observed between them [68-71]. In addition, the production of CAZymes can also be highly strain dependent (see Box $2[66,72]$ ), which would obviously affect the comparisons between species.

The current omics technologies together with recent advances in genetic modification of fungi, such as CRISPR/Cas9 genome editing [73], has opened the door to a much deeper understanding of complex biological processes, such as plant biomass degradation. The high diversity between species and strains puts high challenges on the selection and development of strains and enzyme cocktails for biotechnological applications. The preference of industry for applications using a single fungal strain is opposite to the natural situation, where communities of fungi, bacteria and other organisms fully degrade plant biomass $[74,75]$. Development of novel or improved applications of fungi should therefore not ignore the synergistic value of the enzymes originating from different species [20], and also consider the more challenging, but possibly beneficial effects of fungal co-cultures $[76,77]$.

\section{Acknowledgements}

The Academy of Finland grant no. 308284 to MRM is acknowledged. 


\section{References}

1. Mäkelä, M.R. et al. (2014) Degradation and modification of plant biomass by fungi. In Mycota vol XIII Fungal Genomics ( $2^{\text {nd }}$ edn) (Nowrousian, M. ed), pp. 175-208, Springer-Verlag.

2. de Vries, R.P. and Visser, J. (2001) Aspergillus enzymes involved in degradation of plant cell wall polysaccharides. Microb Mol Biol Rev 65, 497-522.

3. Kubicek, C.P. and Kubicek, E.M. (2016) Enzymatic deconstruction of plant biomass by fungal enzymes. Curr Opin Chem Biol 35, 51-57.

4. Rytioja, J. et al. (2014) Plant-polysaccharide-degrading enzymes from basidiomycetes. Microbiol Mol Biol Rev 78 (4), 614-649.

5. Lombard, V. et al. (2014) The carbohydrate-active enzymes database (CAZy) in 2013. Nucleic Acids Res 42, D490-495.

6. Aspeborg, H. et al. (2012) Evolution, substrate specificity and subfamily classification of glycoside hydrolase family 5 (GH5). BMC Evol Biol 12, 186.

7. Mewis, K. et al. (2016) Dividing the large glycoside hydrolase family 43 into subfamilies: a motivation for detailed enzyme characterization. Appl Environ Microbiol 82 (6), 1686-1692.

8. Stam, M.R. et al. (2006) Dividing the large glycoside hydrolase family 13 into subfamilies: towards improved functional annotations of alpha-amylase-related proteins. Protein Eng Des Sel 19 (12), 555562.

9. Grigoriev, I.V. et al. (2014) MycoCosm portal: gearing up for 1000 fungal genomes. Nucleic Acids Res 42, D699-704.

10. Malgas, S. et al. (2017) Time dependence of enzyme synergism during the degradation of model and natural lignocellulosic substrates. Enzyme Microb Technol 103, 1-11.

11. Van Dyk, J.S. and Pletschke, B.I. (2012) A review of lignocellulose bioconversion using enzymatic hydrolysis and synergistic cooperation between enzymes-factors affecting enzymes, conversion and synergy. Biotechnol Adv 30 (6), 1458-1480.

12. de Vries, R.P. et al. (2000) Synergy between accessory enzymes from Aspergillus in the degradation of plant cell wall polysaccharides. Carbohyd. Res. 327, 401-410.

13. Mäkelä, M.R. et al. (2016) Fungal ligninolytic enzymes and their applications. Microbiol Spectr 4 (6).

14. Champreda, V. et al. (2019) Designing cellulolytic enzyme systems for biorefinery: From nature to application. J Biosci Bioeng 128 (6), 637-654.

15. Goncalves, G.A. et al. (2015) Synergistic effect and application of xylanases as accessory enzymes to enhance the hydrolysis of pretreated bagasse. Enzyme Microb Technol 72, 16-24.

16. Kallioinen, A. et al. (2014) Mixtures of thermostable enzymes show high performance in biomass saccharification. Appl Biochem Biotechnol 173 (5), 1038-1056.

17. Malgas, S. et al. (2017) Formulation of an optimized synergistic enzyme cocktail, HoloMix, for effective degradation of various pre-treated hardwoods. Bioresour Technol 245 (Pt A), 52-65. 18. Neumuller, K.G. et al. (2014) Trichoderma longibrachiatum acetyl xylan esterase 1 enhances hemicellulolytic preparations to degrade corn silage polysaccharides. Bioresource Technol 163, 64-73. 19. Suwannarangsee, S. et al. (2012) Optimisation of synergistic biomass-degrading enzyme systems for efficient rice straw hydrolysis using an experimental mixture design. Bioresour Technol 119, 252-261. 20. van den Brink, J. et al. (2014) Synergistic effect of Aspergillus niger and Trichoderma reesei enzyme sets on the saccharification of wheat straw and sugarcane bagasse. Biotechnol J 9 (10), 1329-1338. 21. Mäkelä, M.R. et al. (2014) Plant biomass degradation by fungi. Fungal Genet Biol 72, 2-9. 22. Baldrian, P. and Valaskova, V. (2008) Degradation of cellulose by basidiomycetous fungi. FEMS Microbiol Rev 32 (3), 501-521.

23. Hatakka, A. and Hammel, K. (2011) Fungal biodegradation of lignocelluloses. In The Mycota, Industrial Applications (Hofrichter, M. ed), pp. 319-340, Springer-Verlag. 
24. Riley, R. et al. (2014) Extensive sampling of basidiomycete genomes demonstrates inadequacy of the white-rot/brown-rot paradigm for wood decay fungi. Proc Natl Acad Sci U S A. 111 (27), 9923-9928. 25. van der Does, H.C. and Rep, M. (2017) Adaptation to the host environment by plant-pathogenic fungi. Annu Rev Phytopathol 55, 427-450.

26. Kämper, J. et al. (2006) Insights from the genome of the biotrophic fungal plant pathogen Ustilago maydis. Nature 444, 97-101.

27. Belmas, E. et al. (2018) Genome sequence of the necrotrophic plant pathogen Alternaria brassicicola Abra43. Genome Announc 6 (6), e01559-17.

28. Padder, B.A. et al. (2016) Transcriptome profiling of the Phaseolus vulgaris - Colletotrichum lindemuthianum pathosystem. PLoS One 11 (11), e0165823.

29. Plett, J.M. and Martin, F. (2011) Blurred boundaries: lifestyle lessons from ectomycorrhizal fungal genomes. Trends Genet 27 (1), 14-22.

30. Dean, R.A. et al. (2005) The genome sequence of the rice blast fungus Magnaporthe grisea. Nature 434, 980-986.

31. Amselem, J. et al. (2011) Genomic analysis of the necrotrophic fungal pathogens Sclerotinia sclerotiorum and Botrytis cinerea. PLoS Genet 7 (8), e1002230.

32. Espagne, E. et al. (2008) The genome sequence of the model ascomycete fungus Podospora anserina. Genome Biol. 9, R77.

33. Martinez, D. et al. (2008) Genome sequencing and analysis of the biomass-degrading fungus Trichoderma reesei (syn. Hypocrea jecorina). Nat Biotechnol 26 (5), 553-560.

34. Bischof, R.H. et al. (2016) Cellulases and beyond: the first 70 years of the enzyme producer Trichoderma reesei. Microb Cell Fact 15 (1), 106.

35. Coutinho, P.M. et al. (2009) Post-genomic insights into the plant polysaccharide degradation potential of Aspergillus nidulans and comparison to Aspergillus niger and Aspergillus oryzae. Fungal Genet Biol 46 Suppl 1, S161-S169.

36. Berka, R.M. et al. (2011) Comparative genomic analysis of the thermophilic biomass-degrading fungi Myceliophthora thermophila and Thielavia terrestris. Nat Biotechnol 29 (10), 922-927.

37. Maitan-Alfenas, G.P. et al. (2016) Characterization and biotechnological application of recombinant xylanases from Aspergillus nidulans. Int J Biol Macromol 91, 60-67.

38. Peng, M. et al. (2017) The draft genome sequence of the ascomycete fungus Penicillium subrubescens reveals a highly enriched content of plant biomass related CAZymes compared to related fungi. J Biotechnol 246, 1-3.

39. Lau, S.K.P. et al. (2017) Talaromyces marneffei genomic, transcriptomic, proteomic and metabolomic studies reveal mechanisms for environmental adaptations and virulence. Toxins (Basel) 9 (6), 192.

40. Vesth, T.C. et al. (2018) Investigation of inter- and intraspecies variation through genome sequencing of Aspergillus section Nigri. Nat Genet 50, 1688-1695.

41. Pel, H.J. et al. (2007) Genome sequencing and analysis of the versatile cell factory Aspergillus niger CBS 513.88. Nat Biotechnol 25 (2), 221-231.

42. Kolbusz, M.A. et al. (2014) Transcriptome and exoproteome analysis of utilization of plant-derived biomass by Myceliophthora thermophila. Fungal Genet Biol 72, 10-20.

43. Rytioja, J. et al. (2017) The molecular response of the white-rot fungus Dichomitus squalens to wood and non-woody biomass as examined by transcriptome and exoproteome analyses. Environ Microbiol 19 (3), 1237-1250.

44. Mäkelä, M.R. et al. (2017) Cultivation of Podospora anserina on soybean hulls results in an efficient enzyme cocktail for plant biomass hydrolysis. N Biotechnol 37, 162-171.

45. Peng, M. et al. (2017) Comparative analysis of basidiomycete transcriptomes reveals a core set of expressed genes encoding plant biomass degrading enzymes. Fung Genet Biol 112, 40-46. 
46. Gaskell, J. et al. (2016) Transcriptome and secretome analyses of the wood decay fungus Wolfiporia cocos support alternative mechanisms of lignocellulose conversion. Appl Environ Microbiol 82 (13), 3979-3987.

47. Gruninger, R.J. et al. (2018) Application of transcriptomics to compare the carbohydrate active enzymes that are expressed by diverse genera of anaerobic fungi to degrade plant cell wall carbohydrates. Front Microbiol 9, 1581.

48. Jurak, E. et al. (2018) Dynamics of the Phanerochaete carnosa transcriptome during growth on aspen and spruce. BMC Genomics 19 (1), 815.

49. Kameshwar, A.K. and Qin, W. (2017) Metadata analysis of Phanerochaete chrysosporium gene expression data identified common CAZymes encoding gene expression profiles involved in cellulose and hemicellulose degradation. Int J Biol Sci 13 (1), 85-99.

50. Vanden Wymelenberg, A. et al. (2009) Transcriptome and secretome analyses of Phanerochaete chrysosporium reveal complex patterns of gene expression. Appl Environ Microbiol 75 (12), 4058-68. 51. Gruben, B.S. et al. (2017) Expression-based clustering of CAZyme-encoding genes of Aspergillus niger. BMC Genomics 18 (1), 900.

52. Benocci, T. et al. (2017) Regulators of plant biomass degradation in ascomycetous fungi. Biotechnol Biofuels 10, 152.

53. Battaglia, E. et al. (2011) Analysis of regulation of pentose utilisation in Aspergillus niger reveals evolutionary adaptations in the Eurotiales. Stud Mycol 69, 31-38.

54. Benocci, T. et al. (2018) ARA1 regulates not only L-arabinose but also D-galactose catabolism in Trichoderma reesei. FEBS Lett 592 (1), 60-70.

55. Klaubauf, S. et al. (2016) A novel L-arabinose-responsive regulator discovered in the rice-blast fungus Pyricularia oryzae (Magnaporthe oryzae). FEBS Lett 590 (4), 550-558.

56. Brown, N.A. et al. (2014) How nutritional status signalling coordinates metabolism and lignocellulolytic enzyme secretion. Fungal Genet Biol 72, 48-63.

57. Seidl, V. et al. (2008) The Hypocrea jecorina (Trichoderma reesei) hypercellulolytic mutant RUT C30 lacks a $85 \mathrm{~kb}$ (29 gene-encoding) region of the wild-type genome. BMC Genomics 9 (1), 327. 58. de Vries, R.P. et al. (1999) CreA modulates the XInR-induced expression on xylose of Aspergillus niger genes involved in xylan degradation. Res Microbiol 150 (4), 281-285.

59. de Vries, R.P. et al. (2002) The Aspergillus niger faeB gene encodes a second feruloyl esterase involved in pectin and xylan degradation, and is specifically induced on aromatic compounds. Biochem. J. 363, 377-386.

60. Mäkelä, M.R. et al. (2018) The fungus Aspergillus niger consumes sugars in a sequential manner that is not mediated by the carbon catabolite repressor CreA. Sci Rep 8, 6655.

61. Hassan, L. et al. (2019) Crosstalk of cellulose and mannan perception pathways leads to inhibition of cellulase production in several filamentous fungi. MBio 10, e00277-19.

62. Kowalczyk, J.E. et al. (2015) Genetic interaction of Aspergillus nidulans galR, $x \ln R$ and araR in regulating $\mathrm{D}$-galactose and $\mathrm{L}$-arabinose release and catabolism gene expression. PLoS One 10 (11), e0143200.

63. Kowalczyk, J.E. et al. (2017) Combinatorial control of gene expression in Aspergillus niger grown on sugar beet pectin. Sci Rep 7, 12356.

64. Yoav, S. et al. (2018) Effects of cre1 modification in the white-rot fungus Pleurotus ostreatus PC9: altering substrate preference during biological pretreatment. Biotechnol Biofuels 11, 212. 65. Todd, R.B. et al. (2014) Prevalence of transcription factors in ascomycete and basidiomycete fungi. BMC Genomics 15, 214.

66. Benoit, I. et al. (2015) Closely related fungi employ diverse enzymatic strategies to degrade plant biomass. Biotechnol Biofuels 8 (1), 107. 
67. Galagan, J.E. et al. (2005) Sequencing of Aspergillus nidulans and comparative analysis with $A$. fumigatus and A. oryzae. Nature 438 (7071), 1105-1115.

68. Alfaro, M. et al. (2014) Comparative analysis of secretomes in basidiomycete fungi. J Proteomics 102, 28-43.

69. Andersen, M.R. et al. (2011) Comparative genomics of citric-acid-producing Aspergillus niger ATCC 1015 versus enzyme-producing CBS 513.88. Genome Res 21 (6), 885-897.

70. Eastwood, D.C. et al. (2011) The plant cell wall-decomposing machinery underlies the functional diversity of forest fungi. Science 333 (6043), 762-765.

71. Wu, W. et al. (2017) Characterization of four endophytic fungi as potential consolidated bioprocessing hosts for conversion of lignocellulose into advanced biofuels. Appl Microbiol Biotechnol 101 (6), 2603-2618.

72. de Vries, R.P. et al. (2017) Comparative genomics reveals high biological diversity and specific adaptations in the industrially and medically important fungal genus Aspergillus. Genome Biol 18 (1), 28. 73. Wang, Q. and Coleman, J.J. (2019) Progress and challenges: Development and implementation of CRISPR/Cas9 technology in filamentous fungi. Comput Struct Biotechnol J 17, 761-769.

74. Baldrian, P. (2017) Microbial activity and the dynamics of ecosystem processes in forest soils. Curr Opin Microbiol 37, 128-134.

75. Lillington, S.P. et al. (2019) Nature's recyclers: anaerobic microbial communities drive crude biomass deconstruction. Curr Opin Biotechnol 62, 38-47.

76. Benoit-Gelber, I. et al. (2017) Mixed colonies of Aspergillus niger and Aspergillus oryzae cooperatively degrading wheat bran. Fungal Genet Biol 102, 31-37.

77. Hu, H.L. et al. (2011) Improved enzyme production by co-cultivation of Aspergillus niger and Aspergillus oryzae with each other and other fungi. Int Biodeter Biodegrad 65, 215-225.

78. Chong, S.L. et al. (2011) The $\alpha$-glucuronidase Agu1 from Schizophyllum commune is a member of a novel glycoside hydrolase family (GH115). Appl Microbiol Biotechnol 90, 1323-1332.

79. Dilokpimol, A. et al. (2018) Fungal glucuronoyl esterases: Genome mining based enzyme discovery and biochemical characterization. N Biotechnol 40 (Pt B), 282-287.

80. Dilokpimol, A. et al. (2018) Fungal feruloyl esterases: Functional validation of genome mining based enzyme discovery including uncharacterized subfamilies. N Biotechnol 41, 9-14.

81. Baker, S.E. (2006) Aspergillus niger genomics: past, present and into the future. Med Mycol 44 Suppl 1, S17-21.

82. Kjærbølling, I. et al. (2019) Friends and foes - A comparative genomics study of 23 Aspergillus species from section Flavi. Nat Comm In press.

83. Kocsube, S. et al. (2016) Aspergillus is monophyletic: Evidence from multiple gene phylogenies and extrolites profiles. Stud Mycol 85, 199-213.

84. van Peij, N.N. et al. (1998) Isolation and analysis of $x \ln R$, encoding a transcriptional activator coordinating xylanolytic expression in Aspergillus niger. Mol Microbiol 27 (1), 131-142.

85. de Vries, R.P. et al. (1999) Differential expression of three $\alpha$-galactosidase genes and a single $\beta$ galactosidase gene from Apergillus niger. Appl Environ Microbiol 65, 2453-2460.

86. Gielkens, M.M. et al. (1999) Two cellobiohydrolase-encoding genes from Aspergillus niger require Dxylose and the xylanolytic transcriptional activator XInR for their expression. Appl Environ Microbiol 65 (10), 4340-4345.

87. Gielkens, M.M.C. et al. (1997) Arabinoxylan degradation by fungi: Characterisation of the arabinoxylan arabinofuranohydrolase encoding genes from Aspergillus niger and Aspergillus tubingensis. Curr Genet 31, 22-29.

88. van Peij, N.N.M.E. et al. (1998) The transcriptional activator XInR regulates both xylanolytic and endoglucanase gene expression in Aspergillus niger. Appl Environ Microbiol 64 (10), 3615-3619. 
89. Calero-Nieto, F. et al. (2007) Role of the transcriptional activator XInR of Fusarium oxysporum in regulation of xylanase genes and virulence. Mol Plant Microbe Interact 20 (8), 977-985.

90. Mach-Aigner, A.R. et al. (2008) Transcriptional regulation of xyr1, encoding the main regulator of the xylanolytic and cellulolytic enzyme system in Hypocrea jecorina. Appl Environ Microbiol 74 (21), 65546562.

91. Marui, J. et al. (2002) A transcriptional activator, AoXInR, controls the expression of genes encoding xylanolytic enzymes in Aspergillus oryzae. Fungal Genet Biol 35 (2), 157-169.

92. Stricker, A.R. et al. (2006) Xyr1 (xylanase regulator 1) regulates both the hydrolytic enzyme system and D-xylose metabolism in Hypocrea jecorina. Eukaryot Cell 5 (12), 2128-2137.

93. Sun, J. et al. (2012) Deciphering transcriptional regulatory mechanisms associated with hemicellulose degradation in Neurospora crassa. Eukaryot Cell 11 (4), 482-493.

94. Klaubauf, S. et al. (2014) Similar is not the same: Differences in the function of the (hemi-)cellulolytic regulator XInR (XIr1/Xyr1) in filamentous fungi. Fungal Genet Biol 72, 73-81.

95. Dos Santos Gomes, A.C. et al. (2019) Myceliophthora thermophila Xyr1 is predominantly involved in xylan degradation and xylose catabolism. Biotechnol Biofuels 12, 220.

96. Battaglia, E. et al. (2011) Carbohydrate-active enzymes from the zygomycete fungus Rhizopus oryzae: a highly specialized approach to carbohydrate degradation depicted at genome level. BMC Genomics 12, 38.

\section{Glossary}

- AraR: transcriptional activator in Eurotiomycetes affecting the expression of arabinanolytic genes and genes of the pentose catabolic pathway

- ARA1: transcriptional activator in Sordariomycetes and Leotiomycetes affecting the expression of arabinanolytic genes and genes of the pentose catabolic pathway

- CAZy: Carbohydrate Active enZYme. This refers to enzymes included in the CAZy database (www.cazy.org) that cleave and/or rearrange glycosidic bonds. Part of the families of this database contain enzymes active on plant biomass.

- CE: carbohydrate esterase. A subsection of the CAZy database containing enzymes that hydrolyze carbohydrate esters.

- CLR1/ClrA: transcriptional activator affecting the expression of cellulolytic genes.

- $\quad$ CLR2/ClrB/ManR: transcriptional activator affecting the expression of cellulolytic and mannanolytic genes.

- CreA/CRE1: main fungal regulator mediating carbon catabolite repression.

- CRISPR/Cas9: Clustered Regularly Interspaced Short Palindromic Repeats/endonuclease. Method to perform genome editing in many organisms, including fungi. 
- GaaR: transcriptional activator affecting the expression of pectinolytic genes and genes of the D-galacturonic acid catabolic pathway.

- GalR: transcriptional activator affecting the expression of genes of the D-galactose oxidoreductive pathway.

- $\mathrm{GH}$ : glycoside hydrolase. A subsection of the CAZy database containing enzymes that use a hydrolytic mechanism.

- PL: polysaccharide lyase. A subsection of the CAZy database containing enzymes that use non-hydrolytic cleavage of glycosidic bonds to degrade polysaccharides.

- RhaR: transcriptional activator affecting the expression of some pectinolytic genes (mainly related to L-rhamnose release) and genes of the L-rhamnose catabolic pathway.

- XInR/XLR1: transcriptional activator expression of (hemi-)cellulolytic genes and some genes of the pentose catabolic pathway.

\section{Text Boxes}

Box 1: Limitation of functional prediction due to lack of a sufficient number of characterized reference enzymes

The inclusion of CAZy annotation in all the fungal genomes hosted in the MycoCosm database [9] has led to many studies comparing the plant biomass degrading ability of fungi based on the number of genes per CAZy family. However, these predictions do not always match the experimentally determined ability. While this could in part be due to the differences in expression of the CAZy genes, another major factor is the sparsity of characterized enzymes in many CAZy families. The percentage of characterized fungal enzymes of the total number of enzymes in CAZy families related to plant biomass degradation is typically low for many families (Fig. I). The characterized members often do not cover the diversity within the family, as revealed by phylogenetic analyses of several families $[78,79]$. This puts further questions on the functional prediction of these candidate enzymes and with that the overall prediction of the degrading ability of a fungus, based on genome annotation. There is an urgent need for more extensive characterization of a well-defined set of fungal reference enzymes for each CAZy family, and the use of genome mining combined with amino acid sequence based phylogeny 
582 offers a perfect starting point for the selection of these reference enzymes [79, 80]. For most

583 CAZy families such phylogenies show distinct clades that may become subfamilies after

584 biochemical characterization.

Figure I. The percentage of characterized fungal CAZymes compared to their total number in 587 the public CAZy database (Adiphol Dilokpimol and Xinxin Li, personal communication). Note that this database does not include most of the fungal genomes present in MycoCosm, but does contain all published characterized enzymes and is therefore a significant overestimation of the percentage of characterized enzymes. $\mathrm{AA}=$ auxiliary activities, $\mathrm{CE}=$ carbohydrate esterase, $\mathrm{GH}$

$591=$ glycoside hydrolase, $\mathrm{PL}=$ polysaccharide lyase .

Box 2: Diversity within the genus Aspergillus

594

Several Aspergillus species (e.g. A. niger, Aspergillus oryzae) have a long history of use in

595 biotechnological applications that include degradation or modification of plant biomass and 596 were among the first fungal species with a sequenced genome [81]. A comparison of the first

597 eight genome-sequenced Aspergilli revealed that the genomic diversity of plant biomass

598 degradation correlates well with their taxonomic relationship [66]. However, proteomic analysis

599 of these species during growth on wheat bran and sugar beet pulp revealed that the enzyme 600 sets they produce differed significantly [66]. Even enzymes for which the gene is present in all 601 species are rarely produced by all species. An expansion of this comparison to 22 Aspergillus 602 species further supported this diversity, suggesting that the different species and even strains 603 of the same species have significant regulatory differences, resulting in the production of 604 different enzyme sets (Fig. II) [72]. Most strikingly, of the two A. niger strains included in this 605 study, one abundantly produced cellulolytic enzymes, while the other produced hardly any. 606 
Figure II. Proteomic response of 17 Aspergilli during growth on sugar beet pulp and wheat bran. The amounts of proteins associated with each enzyme activity were expressed as percentage of the amount of total extracellular proteins present in the cultures [72].

More recently, genomes of all species of the Aspergillus genus have been sequenced enabling a more detailed genomic comparison. Comparative studies of species of two sections, Nigri [40] and Flavi [82], have been published revealing overall similar genome content with respect to CAZy genes related to plant biomass degradation, but in both cases also some species that have a clearly reduced number of these genes. As these differences did not have a clear correlation with growth on plant biomass substrates, this suggests that the phenotypic differences between the species are likely caused at the level of transcriptional regulation. This complete set of fungal genomes of a genus for which the taxonomic relationship of the species has been well established [83], provides a unique opportunity for detailed evolutionary studies in the development of their plant biomass degrading approach. Experimental studies, such as those described above, for a larger number of species is likely to reveal the relevance of individual enzymes in relation to the efficiency of a species to degrade specific plant biomass components.

\section{Box 3: Functional diversity of the (hemi-)cellulolytic regulator $\mathrm{X} \ln R$}

$\mathrm{XInR}$ (also known as XLR1 or XYR1) is one of the most conserved transcriptional activators in filamentous ascomycetes [52]. It was first described as a xylanolytic regulator in A. niger [84], and then shown to also regulate other hemicellulolytic and cellulolytic genes [85-88]. Homologs of the gene have since then also been studied in other species, indicating differences in its set of target genes [89-93]. However, comparison of these studies were difficult due to difference in culture conditions and other experimental approaches. A comparative study of the role of $\mathrm{X} \ln \mathrm{R}$ in five ascomycete species using deletion strains revealed significant diversity with respect to the set of target genes and growth on D-xylose and xylan [94]. In A. niger and A. nidulans growth of the deletion strains was only reduced on xylan but not on D-xylose, while these 
635 mutants of Fusarium graminearum and T. reesei showed reduced growth on both substrates 636 and the deletion strain of Pyricularia (Magnaporthe) oryzae only grew less on D-xylose. This 637 diversity was also reflected in the enzyme activities produced by the strains (Fig. III), which was 638 confirmed by exo-proteomics of the culture filtrates demonstrating distinct enzyme sets that 639 decreased in the deletion mutants of the species [94]. A later study in M. thermophila, using the 640 same experimental conditions, showed that the homolog in this species (Xyr1) mainly regulated 641 xylanolytic genes and genes of pentose catabolism, but only to a small extent cellulolytic and 642 other hemicellulolytic genes [95].

644 Figure III. Relative activity of $x \ln R$ deletion strains after growth on xylan [94]. CBH = 645 cellobiohydrolase, $B X L=\beta$-xylosidase, $B G L=\beta$-glucosidase,$A B F=\alpha$-arabinofuranosidase.

Figure Legends

649 Figure 1. Comparison of growth of three ascomycete fungi on xylan and pectin, and their 650 monomeric components (www.fung-growth.org), and the number of putative xylanolytic and 651 pectinolytic genes in their genome $[31,32,96]$. The CAZy families that were taken into account 652 in this comparison are listed.

654 Figure 2. Model for the indirect interaction between regulators involved in plant biomass 655 degradation in A. niger, based on [51]. The transcriptional activators facilitate the release of 656 various monomers from plant biomass (indicated by thin arrows). These monomers themselves 657 result in activations of specific regulators (indicated by thick arrows). CreA has an overruling 658 effect in that high concentrations of any of the monomers will repress plant biomass 659 degradation. 
Table 1. Comparison of the number of genes in selected CAZy glycoside hydrolase (GH) families related to plant biomass degradation in species of the same genus. Data was based on the CAZy annotation tables of published genomes in MycoCosm [9]. Expanded gene numbers are in bold.

Species

$\begin{array}{lllllllllllllllllllllllllllll}G H & 1 & 2 & 3 & 5 & 6 & 7 & 10 & 11 & 12 & 26 & 27 & 28 & 29 & 35 & 36 & 39 & 43 & 45 & 51 & 53 & 54 & 62 & 67 & 74 & 78 & 93 & 95 & 115\end{array}$

Aspergillus aculeatus

Aspergillus brasiliensis

Aspergillus carbonarius

Aspergillus clavatus

Aspergillus fischeri

Aspergillus flavus

Aspergillus fumigatus

Aspergillus luchuensis

Aspergillus nidulans

Aspergillus niger

Aspergillus oryzae

Aspergillus sydowii

Aspergillus terreus

Aspergillus tubingensis

Aspergillus versicolor

Aspergillus wentii

Meliniomyces bicolor

Meliniomyces variabilis

$\begin{array}{llllllllllllll}3 & 6 & 17 & 12 & 1 & 2 & 2 & 3 & 4 & 1 & 4 & 19 & 1 & 4\end{array}$

$\begin{array}{ll}0 & 15\end{array}$

5

1

11

6

$\begin{array}{lll}2 & 3 & 0\end{array}$

$\begin{array}{lllllllllllllllllllllllllllllllllll}3 & 6 & 21 & 11 & 2 & 2 & 3 & 4 & 5 & 1 & 4 & 20 & 1 & 6 & 2 & 0 & 11 & 0 & 4 & 1 & 1 & 1 & 1 & 1 & 8 & 1 & 2 & 0\end{array}$

$\begin{array}{lllllllllllllllllllllllllllll}3 & 5 & 16 & 9 & 1 & 2 & 0 & 2 & 3 & 0 & 3 & 19 & 1 & 4 & 1 & 0 & 13 & 0 & 3 & 1 & 1 & 1 & 1 & 0 & 5 & 1 & 2 & 0\end{array}$

$\begin{array}{rrrrrrrrrrrrrrrrrrrrrrrrrrrrrrr}4 & 3 & 12 & 9 & \mathbf{2} & \mathbf{4} & 2 & 3 & 3 & 0 & 3 & 3 & 0 & 3 & 3 & 1 & 13 & 0 & 3 & 0 & 1 & 2 & 1 & 1 & 0 & 1 & 1 & 1 \\ \mathbf{5} & 6 & 19 & 16 & \mathbf{2} & \mathbf{5} & \mathbf{4} & \mathbf{4} & 5 & 0 & 5 & 13 & 0 & 5 & 3 & 1 & 20 & 1 & 2 & 1 & 1 & \mathbf{3} & 1 & 2 & 7 & 3 & 2 & 1\end{array}$

$\begin{array}{rlllllllllllllllllllllllllllll}5 & 6 & 19 & 16 & \mathbf{2} & \mathbf{5} & \mathbf{4} & \mathbf{4} & 5 & 0 & 5 & 13 & 0 & 5 & 3 & 1 & 20 & 1 & 2 & 1 & 1 & \mathbf{3} & 1 & 2 & 7 & \mathbf{3} & 2 & 1\end{array}$

$\begin{array}{lllllllllllllllllllllllllllll}3 & 8 & 24 & 15 & 1 & 3 & \mathbf{4} & \mathbf{4} & 5 & 1 & 3 & 21 & 0 & \mathbf{8} & 3 & 0 & 21 & 1 & 4 & \mathbf{2} & 1 & 2 & 1 & 0 & \mathbf{1 2} & \mathbf{3} & 3 & \mathbf{3}\end{array}$

$\begin{array}{llllllllllllllllllllllllllllll}5 & 6 & 18 & 14 & 1 & 4 & 4 & 3 & 4 & 0 & 5 & 12 & 0 & 5 & 3 & 1 & 18 & 1 & 2 & 1 & 1 & 2 & 1 & \mathbf{2} & 5 & \mathbf{3} & 2 & 1\end{array}$

$\begin{array}{lllllllllllllllllllllllllllllll}2 & 6 & 16 & 9 & 2 & 2 & 2 & 4 & 4 & 1 & 4 & 19 & 1 & 5 & 2 & 0 & 11 & 0 & 4 & 2 & 1 & 1 & 1 & 1 & 7 & 1 & 2 & 0\end{array}$

$\begin{array}{lllllllllllllllllllllllllllll}4 & 10 & 21 & 16 & 2 & 3 & 3 & 2 & 1 & 3 & 4 & 11 & 0 & 4 & 4 & 2 & 19 & 1 & 2 & 1 & 1 & 2 & 1 & 2 & 9 & 2 & 3 & 1\end{array}$

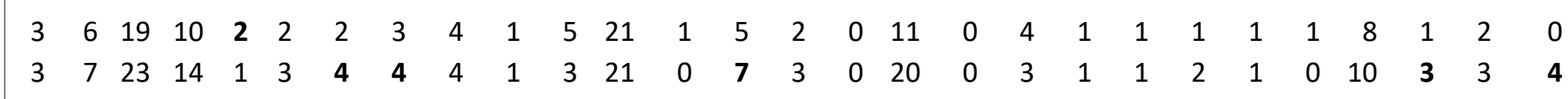

$\begin{array}{lllllllllllllllllllllllllllll}3 & 13 & 29 & 12 & 1 & 3 & 2 & 3 & 2 & 1 & 6 & 11 & 0 & 4 & 5 & 0 & 25 & 1 & 2 & 1 & 1 & 2 & 1 & 1 & 9 & 3 & 3 & 3\end{array}$

$\begin{array}{lllllllllllrllllllllllllllllll}3 & 10 & 21 & 18 & 2 & 4 & 4 & 2 & 6 & 0 & 5 & 8 & 2 & 4 & 4 & 2 & 21 & 0 & 4 & 1 & 1 & 3 & 2 & 1 & 4 & 4 & 3 & 2\end{array}$

$\begin{array}{llllllllllllllllllllllllllllll}3 & 6 & 18 & 10 & 2 & 2 & 2 & 4 & 4 & 2 & 4 & 19 & 1 & 5 & 2 & 0 & 11 & 0 & 4 & 2 & 1 & 1 & 1 & 1 & 7 & 1 & 2 & 0\end{array}$

$\begin{array}{lllllllllllllllllllllllllllll}5 & 13 & 28 & 14 & 1 & 3 & 4 & 3 & 3 & 1 & \mathbf{6} & 13 & 0 & 6 & 5 & 0 & \mathbf{2 7} & 1 & 2 & \mathbf{3} & 1 & 2 & 1 & 1 & \mathbf{1 1} & \mathbf{4} & \mathbf{4} & \mathbf{6}\end{array}$

Penicillium brevicompactum

Penicillium canescens

Penicillium chrysogenum

Penicillium rubens

Penicillium digitatum

Penicillium expansum

Penicillium oxalicum

Penicillium subrubescens

\begin{tabular}{rrrrrrrrrrrrrrrrrrrrrrrrrrrr}
3 & 5 & 16 & 11 & 1 & 3 & 4 & 1 & 3 & 0 & 2 & 12 & 1 & 4 & 2 & 0 & 14 & 0 & 3 & 1 & 0 & 1 & 0 & 0 & 5 & 4 & 1 & 1 \\
\hline 4 & 6 & 17 & 24 & 2 & 5 & 4 & 5 & 5 & 0 & 10 & 18 & 1 & 5 & 3 & 1 & 15 & 3 & 5 & 2 & 1 & 1 & 1 & 2 & 9 & 4 & 4 & 3
\end{tabular}

Phanerochaete carnosa

\begin{tabular}{rrrrrrrrrrrrrrrrrrrrrrrrrrrr}
5 & $\mathbf{1 2}$ & $\mathbf{2 8}$ & $\mathbf{3 4}$ & $\mathbf{4}$ & $\mathbf{9}$ & 5 & $\mathbf{8}$ & $\mathbf{4}$ & $\mathbf{2}$ & 12 & 23 & $\mathbf{1}$ & 6 & $\mathbf{4}$ & $\mathbf{5}$ & 16 & $\mathbf{4}$ & $\mathbf{9}$ & $\mathbf{3}$ & $\mathbf{3}$ & $\mathbf{2}$ & $\mathbf{2}$ & $\mathbf{2}$ & $\mathbf{1 4}$ & $\mathbf{9}$ & $\mathbf{3}$ & 3 \\
\hline 4 & 7 & 20 & 15 & 1 & 2 & 2 & 1 & 3 & 0 & 3 & 12 & 1 & 4 & 3 & 0 & 15 & 0 & 3 & 1 & 1 & 2 & 1 & 0 & $\mathbf{9}$ & 2 & 1 & 1 \\
6 & 9 & 22 & 17 & 1 & 3 & $\mathbf{4}$ & 1 & 4 & 0 & 4 & 15 & 1 & $\mathbf{7}$ & 2 & 1 & 21 & 0 & 3 & 1 & 1 & 3 & 1 & 0 & $\mathbf{9}$ & $\mathbf{4}$ & $\mathbf{2}$ & 1 \\
3 & 7 & 16 & 14 & 1 & 2 & 3 & 1 & 4 & 1 & 2 & 5 & 0 & 4 & 2 & 2 & 13 & 0 & 3 & 1 & 1 & 1 & 1 & 0 & 5 & 2 & 1 & 0 \\
3 & 6 & 18 & 13 & 1 & 2 & 3 & 1 & 3 & 1 & 2 & 5 & 0 & 4 & 2 & 2 & 14 & 0 & 3 & 1 & 1 & 1 & 1 & 0 & 5 & 2 & 1 & 0 \\
3 & 4 & 15 & 8 & 1 & 2 & 1 & 1 & 2 & 0 & 0 & 8 & 0 & 4 & 2 & 0 & 3 & 0 & 2 & 1 & 1 & 0 & 1 & 0 & 4 & 2 & 1 & 0 \\
6 & 10 & 25 & 13 & 1 & 2 & 3 & 1 & 4 & 1 & 0 & 9 & 0 & 4 & 2 & 0 & 9 & 0 & 4 & 1 & 1 & 0 & 1 & 0 & 7 & 2 & 1 & 0 \\
4 & 6 & 14 & 13 & 1 & 3 & 3 & $\mathbf{5}$ & 3 & 1 & 2 & 11 & 0 & 3 & 1 & 0 & 14 & 1 & 3 & 1 & 1 & 2 & 1 & 0 & 4 & 3 & 1 & 0 \\
6 & 11 & 25 & $\mathbf{2 1}$ & $\mathbf{2}$ & $\mathbf{4}$ & 3 & $\mathbf{7}$ & $\mathbf{8}$ & 0 & $\mathbf{7}$ & $\mathbf{2 2}$ & $\mathbf{4}$ & $\mathbf{6}$ & $\mathbf{7}$ & $\mathbf{4}$ & $\mathbf{3 0}$ & 1 & $\mathbf{6}$ & 1 & $\mathbf{4}$ & $\mathbf{4}$ & $\mathbf{4}$ & 0 & $\mathbf{1 1}$ & 3 & $\mathbf{2}$ & $\mathbf{3}$ \\
\hline 2 & 2 & 11 & $\mathbf{2 4}$ & 1 & 5 & 5 & 1 & 3 & 0 & 3 & 4 & 0 & 4 & 0 & 0 & 4 & 1 & 2 & 1 & 0 & 0 & 0 & 2 & 1 & 0 & 1 & 1
\end{tabular}


Phanerochaete chrysosporium Talaromyces aculeatus Talaromyces funiculosus Talaromyces marneffei Talaromyces stipitatus

Trichoderma atroviride Trichoderma harzianum Trichoderma reesei Trichoderma virens

\begin{tabular}{|rrrrrrrrrrrrrrrrrrrrrrrrrrrrr}
2 & 2 & 10 & 19 & 1 & $\mathbf{8}$ & 6 & 1 & 2 & 0 & 3 & 5 & 0 & 3 & 0 & 0 & 4 & 2 & 2 & 1 & 0 & 0 & 0 & $\mathbf{4}$ & 1 & 0 & 1 & 1 \\
\hline $\mathbf{8}$ & $\mathbf{9}$ & $\mathbf{3 3}$ & $\mathbf{1 5}$ & 1 & 3 & 2 & $\mathbf{7}$ & $\mathbf{7}$ & 1 & 5 & $\mathbf{2 1}$ & 3 & $\mathbf{1 3}$ & $\mathbf{7}$ & $\mathbf{9}$ & $\mathbf{2 3}$ & 2 & $\mathbf{6}$ & 1 & 6 & $\mathbf{4}$ & 2 & 1 & $\mathbf{1 9}$ & $\mathbf{5}$ & $\mathbf{4}$ & 1 \\
4 & $\mathbf{9}$ & $\mathbf{3 1}$ & $\mathbf{1 6}$ & 1 & $\mathbf{4}$ & 1 & $\mathbf{8}$ & $\mathbf{6}$ & 1 & $\mathbf{9}$ & $\mathbf{2 1}$ & 3 & 5 & $\mathbf{1 0}$ & 4 & $\mathbf{2 4}$ & 2 & $\mathbf{6}$ & 1 & $\mathbf{9}$ & $\mathbf{4}$ & $\mathbf{4}$ & 1 & $\mathbf{1 7}$ & $\mathbf{5}$ & $\mathbf{5}$ & $\mathbf{3}$ \\
3 & 6 & 14 & 8 & 1 & 2 & 1 & 3 & 3 & 0 & 2 & 8 & 0 & 4 & 1 & 1 & $\mathbf{7}$ & 2 & 1 & 1 & 4 & 2 & 2 & 1 & 2 & 2 & 1 & 0 \\
3 & 5 & 24 & 11 & 1 & 2 & 2 & 4 & 3 & 0 & 4 & 8 & 2 & 4 & 3 & 2 & 11 & 2 & 2 & 1 & 3 & 2 & 3 & 1 & 2 & 3 & 2 & 1 \\
\hline $\mathbf{4}$ & 10 & 15 & 11 & 1 & 2 & 1 & 4 & 3 & 0 & 9 & 6 & 0 & 1 & 2 & 2 & $\mathbf{6}$ & 1 & $\mathbf{1}$ & 0 & 2 & 2 & 2 & 1 & $\mathbf{3}$ & $\mathbf{3}$ & 4 & 1 \\
$\mathbf{4}$ & $\mathbf{1 3}$ & $\mathbf{1 8}$ & 12 & 1 & 2 & 2 & 4 & 3 & $\mathbf{2}$ & 9 & 6 & 0 & 1 & 2 & $\mathbf{3}$ & 4 & $\mathbf{3}$ & 0 & 0 & 2 & 2 & 2 & 1 & 2 & 2 & 5 & 1 \\
2 & 8 & 13 & 8 & 1 & 2 & 1 & 3 & 2 & 0 & 8 & 4 & 0 & 1 & 2 & 1 & 2 & 1 & 0 & 0 & 2 & 1 & 1 & 1 & 1 & 0 & 4 & 1 \\
2 & 11 & $\mathbf{1 7}$ & 12 & 1 & 2 & 2 & 4 & $\mathbf{4}$ & $\mathbf{2}$ & 11 & 6 & 0 & 1 & 2 & 1 & 3 & 2 & 0 & 0 & 2 & $\mathbf{3}$ & 2 & 1 & $\mathbf{3}$ & 1 & 4 & 1
\end{tabular}

